\title{
Response to Commentaries on "A Neurobiological Model for Near-Death Experiences"
}

\author{
Juan C. Saavedra-Aguilar, M.D. \\ Juan S. Gómez-Jeria, Lic.Q. \\ University of Chile
}

We are pleased and grateful for the interest that our work has elicited in the preceding commentaries, and in the following pages we present pertinent responses.

Our neurobiological "model" for near-death experiences (NDEs) was intended to be a preliminary work to be completed or modified by others, and may be considered a scientific hypothesis rather than a model. It fulfills the following criteria for scientific hypotheses: it is formally structured and not semantically empty, it is rooted in previous scientific knowledge, and it can be empirically tested by scientific procedures (Bunge, 1979).

This last condition deserves commentary. From the strict scientific point of view, NDEs could be provoked in some individuals in order to measure the different biochemical and electrical brain changes correlated with them. However, accepted and sound ethical principles do not permit that. With the current use of noninvasive brain imaging systems, that impediment could be surmounted by the constant monitor-

This work has received financial support from FONDECYT (Project 1111-88). The authors also acknowledge the helpful comments of Dr. Bruce Cassels.

Dr. Saavedra-Aguilar is Assistant Professor in the Department of Neurological Sciences in the Faculty of Medicine, University of Chile. Lic. Gómez-Jeria is Assistant Professor in the Faculty of Sciences at the University of Chile. Requests for reprints should be addressed to Dr. Saavedra-Aguilar at the Universidad de Chile, Facultad de Medicina, Departamento de Ciencias Neurologicas, Av. Jose Miguel Infante 555, Santiago, Chile; or to Lic. Gómez-Jeria at the Universidad de Chile, Facultad de Ciencias, Departmento de Quimica, Casilla 653, Santiago, Chile. 
ing of subjects who spontaneously present in cardiac arrest. In that sense, Glen Gabbard and Stuart Twemlow's assertion that our model is too nonspecific to be useful is not valid.

Out hypothesis was constructed in keeping with the scientific method. In that sense, and considering Patricia Smith Churchland's statement that "there is a complete absence of any positive evidence for a non-physical substance, a lack of any genuine explanation of how the two substances (mind and brain) might interact, and there simply is no distinctly dualistic methodology or testable theory" (Churchland, 1988 , p. 305), we have discarded from our discussion any notions like astral bodies, souls, mysterious vapors, paranormal entities, karmic illusions, rebirth, or reincarnation. Melvin Morse raises a very important problem for the supporters of these beliefs when he points out the contradiction that "the spirit rises out of the body leaving the brain behind, but somehow still incorporating neuronal functions such as sight, hearing, and proprioception."

The use of the scientific method to construct hypotheses contrains the verbal system so as to exclude personal beliefs and superstitions. Ernst Rodin's comment on the role of our verbal systems in the construction of our hypothesis does not take into account that constraint. Although like any scientific communication, our paper uses written language, we believe his commentary is epistemologically erroneous. Also, our work is not a mere revision or embellishment of previous works, but a new scientific creative hypothesis (Storr, 1986).

Regarding Rodin's deja vu problems, which can be interpreted in various interesting ways, we did not cite his paper because we had a jamais vu problem at that time (Rodin, 1980). We read his paper again and still consider it to be an essay, neither a model nor a hypothesis. In contrast, we regard Daniel Carr's pioneering work as a serious scientific attempt to explain NDEs in detail (Carr, 1982).

Also, our inclusion in our hypothesis of "epileptic-like" phenomena, neurotransmitter activity, and the effects of hypoxia is not mixing concepts, as Russell Noyes objected, but working with different explanatory levels, an essential aspect of this kind of work. Clearly, we may formulate scientific hypotheses to explain NDEs that are based on brain functioning at different levels, including the atomic, molecular, biochemical, and neurophysiological. From a practical point of view some of these levels are still not suitable to work with; quantum mechanical explanations for NDEs are not forthcoming in the near future, but they are theoretically possible!

In spite of the fact that we presented an operational definition for NDEs (Greyson and Stevenson, 1980), Noyes demands that we define 
just what we mean by an NDE. It is noteworthy that Richard Blacher employs several definitions. This touches a central point for the scientific investigation of this kind of phenomenon: a formal definition and diagnostic criteria for NDEs do not exist. While it seems highly necessary to design formal and well-structured tests that use stringent criteria to detect NDEs, this area is in its infancy. The question of exactly what are the "symptoms" of NDEs has not been unambiguously answered.

Given their variety and the way they appear grouped in different studies, we wonder whether all the cases reported as NDEs are such. The same can be said about out-of-body experiences: some subjects "feel" as if they were out of their bodies without watching themselves, others "see" themselves as if from out of their bodies, while still others see their "double" but do not feel out of their own bodies.

Without strict definitions supported by appropriate experiments, it seems very difficult to present a complete hypothesis covering absolutely all the experiential phenomena described as NDEs. This limitation was explicitly stated in our paper. The phenomenological overlap that NDEs present with other phenomena is not unique; another example of this phenomenological overlap can be found in an article on multiple personality and obsessive-compulsive disorder (Ross and Anderson, 1988).

We agree with James McHarg that perceptual alterations, such as the noises reported in the early stages of NDEs, could arise from other regions of the brain. We emphasized the organ of Corti involvement to suggest that even peripheral organs could contribute to NDE phenomenology. This stresses the fact that NDE symptoms cannot be strictly localized in specific parts of the brain.

Dysmnesic phenomena are essential to our hypothesis, permitting the storage of fragmented emotionally important features of the episode by memory mechanisms. Amnesia, by definition, prevents the recollection of the episode. In any case, McHarg's suggestion that dysmnesia contributes to the sensation of peace is a very interesting one, and merits consideration in future refinements of NDE pathophysiology.

Brain stress, a term McHarg found imprecise, could be considered to be any reversible and sudden change in the brain's normal functioning. This can be produced by, for example, cardiac arrest, car accidents, torture, or kidnapping, but not, as Blacher noted, by a bullet passing one inch away from the subject's head without his or her knowledge! Stress does not necessarily imply a negative psychological reaction, like anxiety, or noticeable autonomic changes. On the contrary, some 
stressed subjects can present with mental calmness and relaxation, as Gabbard and Twemlow noted. With that understanding, we think that our hypothesis is in agreement with all relevant scientific data.

Regarding Noyes's assertion that if NDEs are abnormal like temporal lobe epilepsy they are therefore "without adaptive potential or meaning," we suggested only that NDEs arise from the abnormal functioning of some portions of the brain. Whether or not NDEs have an adaptive value is beyond the scope of our work. Nevertheless, the expression "adaptive value" needs to be analyzed. Does schizophrenia have adaptive value? Does temporal lobe epilepsy? It would seem better to consider all these conditions as ontogenetic responses to a given situation.

We think that Noyes also misinterpreted us in regard to anoxia. We did not say that NDEs, by definition, involved anoxia, but only a sudden and stressful situation coming from the diminished quantity of cerebral blood flow and oxygen, or hypoxia. In any case, we do not see how stress per se can produce anoxia.

We did not localize the focus of all NDE phenomena in the temporal lobe. We suggested that NDEs correspond to a dysfunction of a cortical and subcortical system in which limbic structures play a fundamental role. Gabbard and Twemlow commented that since all mental phenomena are connected to limbic structure, the suggestion that NDEs originate in that portion of the brain is of limited heuristic value.

That comment is not correct. On the contrary, limbic structures are one of two sites of convergence of information from the external world and the internal milieu. They connect data from the external world with instinctive drives, acting as a kind of "emotional selection unit" crucial to the proper balance between instinctive programmed drives and actual objective situations, providing the adequate motivational bias. The other site of convergence involves the prefrontal dorsolateral and basomedial association areas, which also function as a kind of selection unit for appropriate behavior, integrating the motivational information coming from high-order heteromodal cortices.

The diverse evidence presented in our paper and elsewhere seems more related to a dysfunction of the limbic selection unit because of its importance in stressful and life-threatening situations, which generally involve alterations of consciousness. The prefrontal selection unit functions in situations with a normal level of consciousness and is probably more important for the recollection and later verbal description of the episode. This simplified scheme has heuristic value and is not a sweeping generalization because it is rooted in solid functional and anatomical evidence (Goldman-Rakic, 1984; Mesulam, 1986; Benson, 1987). 
We think that the neurological mechanisms we outlined for NDEs, the verbal system and episodic memory, also explain the contents of NDEs, which belong for the moment to the psychologcial domain. Personally, we think that the notion of separate domains makes the problem of understanding human consciousness more difficult. All domains must eventually be integrated with neurophysiological knowledge.

Gabbard and Twemlow felt that we misinterpreted the conclusions of Jerome Tobacyk and Thomas Mitchell (1987) to fit our hypothesis. We suggested that the out-of-body experience (OBE) seemed to correlate with some personality variables, while Gabbard and Twemlow argued that Tobacyk and Mitchell did not find any significant differences among OBE reporters and nonreporters. However, Tobacyk and Mitchell wrote that "reporters of out-of-body experiences did show significantly greater belief in psi, precognition, spiritualism, and witchcraft than did nonreporters" (Tobacyk and Mitchell, 1987, p. 369). These findings could mean that cultural bias could lead some OBE reporters to interpret their "uncommon sensations" as being OBEs.

Carr's comments are very gratifying, but we do not fully agree with his assertion that complex perceptual processes and illnesses like depression, anxiety, and schizophrenia are well understood. In that regard, we feel that NDEs, because of their nature, suffer only slightly more from a lack of understanding. We hope our work has made them a little more understandable.

Morse's sophisticated contribution helped us very much towards a further understanding of this complex subject. We believe this kind of approach is the only way of dealing with the problem, or pseudoproblem, of brain-mind interaction.

The value of understanding the NDE's biological basis is a very important issue for the essential question of natural philosophy: what are the bases of human consciousness? Furthermore, even if the biological study of NDEs had no practical value, it would be an example of the essence of science, that is, knowledge per se. Carr's final quotation from Churchland elegantly summarizes the epistemological value of our work in making sense of ourselves.

Blacher's comparison between NDEs and dreaming is very stimulating. In this regard, we may note that some subjects report phenomenological descriptions of episodes occurring during rapid eye movement (REM) sleep that are almost identical to NDEs (Van Eeden, 1913). Whether or not NDE-like phenomena in REM sleep can be integrated into our hypothesis is a question that must still await an answer. Still, during REM sleep there are conspicuous changes in some neuromodulators such as cortisol, acetylcholine, and growth hormone 
(Weitzman, 1982; Gillin, Sitaram, and Mendelson, 1982). REM sleep could be considered as a kind of weak physiological brain stress, in contrast to the normal waking state.

Rodin's scientific observations necessitate several comments. First, the fact that he never came across NDE phenomenology in epileptic patients is in complete agreement with our hypothesis. NDErs have a previously functionally intact brain, which is not the case with epileptic subjects. This difference also explains why electrical stimulation of the brains of epileptic patients fails to produce a full NDE.

Second, in relation to occipital lobe discharges, Rodin confuses the physiophathology and phenomenology of classical migraine with the physiopathology of epilepsy. Third, there is a difference between epileptic seizure discharges and epileptiform activity.

Fourth, Rodin's own assertion that "whatever goes on in the blood in regard to ACTH or endogenous opiates may bear very little relation to what happens in discrete areas of the brain," renders irrelevant his discussion of the work of Dennis Kelly and of Crawford Clark, Joseph Yang, and Malvin Janal.

Fifth, we suggest that rats cannot hallucinate or have NDEs as humans do because their brain organization is very different. For example, it has shown that a novel class of acetylcholinesterase-rich neurons represents a unique human adaptation and provides the neuroanatomical substrate for human mental phenomena (Mesulam and Geula, 1988). Also, the unique organization of the human prefrontal lobe is not shared by rats (Goldman-Rakic, 1984, 1988).

Sixth, Rodin's doubts about the appearance of hallucinations following an acute decrease of acetylcholine levels, such as following a central acetylcholinergic blocking agent, are not shared by others working in this area. His example of Alzheimer's disease is marginal because that disease arises gradually and acetylcholine is not the only neurotransmitter involved.

Seventh, the cerebellar involvement in anoxia is unrelated to our discussion, and eighth, the notion that neurophysiology and neurochemistry give isolated insights does not necessarily lead to speculations. In relation to will and free will, we suggest the reading of a fundamental paper by Benjamin Libet, Curtis Gleason, Elwood Wright, and Dennis Pearl (1983).

Finally, it seems necessary now to include the possible role of sigma and excitatory aminoacid receptors (kainate, quisqualate, and glutamate) in NDE phenomenology. Sigma receptors are involved in a variety of mental disturbances, such as hallucinations and psychotic ideation (Saavedra-Aguilar, Morales-Lagos, and Gómez-Jeria, 1989). 
On the other hand, psychological stress leads to an increase in plasma levels of some steroids such as cortisol and corticosterone, which increase dopaminergic activity (Meyerhoff, Oleshansky, and Mougey, 1988; Wolkowitz, Sutton, Koulu, Labarca, Wilkinson, Doran, Hauger, Pickar, and Crawley, 1986). Since sigma receptors have high affinity for some steroids, they could play a role in some stress-induced mental phenomena like NDEs (Su, London, and Jaffe, 1988).

\section{References}

Benson, D. F. (1987). The frontal lobe. New York, NY: Macmillan.

Bunge, M. (1979). La investigacion cientifica. Barcelona, Spain: Ariel.

Carr, D. B. (1982). Pathophysiology of stress-induced limbic lobe dysfunction: An hypothesis for NDEs. Anabiosis: The Journal of Near-Death Studies, 2, 75-89.

Churchland, P. S. (1988). The significance of neurosciences for philosophy. Trends in Neurosciences, 11, 304-307.

Gillin, J. C., Sitaram, N., and Mendelson, W. B. (1982). Acetylcholine, sleep and depression. Human Neurobiology, 1, 211-219.

Goldman-Rakic, P. S. (1984). The frontal lobes: Uncharted provinces of the brain. Trends in Neurosciences, 7, 425-429.

Goldman-Rakic, P. S. (1988). Topography of cognition: Parallel distributed networks in primate association cortex. Annual Review of Neuroscience, 11, 137-156.

Greyson, B., and Stevenson, I. (1980). The phenomenology of near-death experiences. American Journal of Psychiatry, 137, 1193-1196.

Libet, B., Gleason, C. A., Wright, E. W., and Pearl, D. K. (1983). Time of conscious intention to act in relation to onset of cerebral activity (readiness-potential). Brain, $106,623-642$.

Mesulam, M. M. (1986). Principles of behavioral neurology. Philadelphia, PA: F. A. Davis.

Mesulam, M. M., and Geula, C. (1988). Acetylcholinesterase-rich pyramidal neurons in the human neocortex: Absence at birth, development during the life span, and dissolution in Alzheimer's disease. Annals of Neurology, 24, 765-773.

Meyerhoff, J. L., Oleshansky, M. A., and Mougey, E. H. (1988). Psychologic stress increases plasma levels of prolactin, cortisol and POMC-derived peptides in man. Psychosomatic Medicine, 50, 295-303.

Ross, C. A., and Anderson, G. (1988). Phenomenological overlap of multiple personality disorder and obsessive-compulsive disorder. Journal of Nervous and Mental Disease, 176, 295-299.

Rodin, E. A. (1980). The reality of death experiences. Journal of Nervous and Mental Disease, 168, 259-263.

Saavedra-Aguilar, J. C., Morales-Lagos, D., and Gómez-Jeria, J. S. (1989). Sigma and PCP/NMDA receptors: Their physiopathological role in some psychotic pictures and their pharmacological treatment. Revista Chilena de Neuro-Psiquiatria, 27, 41-48.

Storr, A. (1986). The dynamics of creation. New York, NY: Penguin Books.

Su, T. P., London, E. D., and Jaffe, J. H. (1988). Steroid binding to sigma receptors suggests a link between endocrine, nervous and immune systems. Science, 240, 219-221.

Tobacyk, J. J., and Mitchell, T. P. (1987). The out-of-body experience and personality adjustment. Journal of Nervous and Mental Disease, 175, 367-370.

Van Eeden, F. (1913). A study of dreams. Proceedings of the Society for Psychical Research, 26, 431-461. 
Weitzman, E. D. (1982). Chronobiology of man. Human Neurobiology, 1, 173-183.

Wolkowitz, O., Sutton, M., Koulu, M., Labarca, R., Wilkinson, L., Doran, A., Hauger, R., Pickar, D., and Crawley, J. (1986). Chronic corticosterone administration in rats: Behavioral and biochemical evidence of increased central dopaminergic activity. European Journal of Pharmacology, 122, 329-338. 